\title{
Exploratory Data Analysis and Offence Prediction
}

\author{
VivekGauravSingh $^{\mathrm{a}}$, Shwetank Singh Prashun ${ }^{\mathrm{b}}$, R.Brindha $^{\mathrm{c}}$ \\ ${ }^{\mathrm{a}, \mathrm{b}}$ Department of Computer Science Engineering, SRMIST(Kattankulathur),Chennai, India
}

${ }^{\mathrm{c}}$ AssistantProfessor,Department of Computer Science

Engineering,SRMIST(Kattankulathur),Chennai,India

ㄹvv8567@ srmist.edu.in, ${ }^{\text {b }}$ sm6818@ @ srmist.edu.in,, brindhar1@ srmist.edu.in

\begin{abstract}
Big data is a part of data science that pinpoint different ways to diagnosis, systematically withdraw facts from informational collections that are excessively enormous or complex to be managed by customary information handling application software. Big Data Analytics(BDA) is a specific tactic for breaking down and recognizing assorted examples, kindred, and patterns inside a massive volume in order. Big data analytics (BDA) is a meticulous approach to data analysing and recognising unique layers, connections, and trends ina gigantic volume of data. We apply BDA to illegitimate information collected in this paper, where preliminary data analysis was conducted for visual analysis and trend prediction. Following statistical analysis and visualisation, some incredibly interesting facts and patterns emerge from illegal data in INDIAN states i.e. (Uttar Pradesh, New Delhi, Goa). The prognostic results demonstrate that Kerasstateful LSTM execute enhanced than neural network models. These capable outcomes will allow police departments and law enforcement agencies to better understand crime problems and gain insights that will allow them to schedule activities, predict the likelihood of incidents, efficiently allocate resources, and optimise decision making.
\end{abstract}

Main Phrases: Huge information investigation, information mining, and information perception.

\section{INTRODUCTION}

Information Mining is the strategy which remembers evaluating and analytical massive previous list for teach to induce novel data which might be key to the association. The extraction of novel data is guage utilizing the current datasets. Numerous weaving machines examination and figure in information mining had been executed. Be that as it may, innumerable few difficult work has finished in the criminal science glade. Various few have taken difficult work for assessing the data every one of these weaving machines. The police headquarters and other practically equivalent to unlawful equity organizations handle numerous cumbersome data sets of data which can be worn to visualize or dissect the illegal developments and illegal clamor association in the way of life. The illicits can likewise be imagined dependent on the offense information.

Customarily addressing offenses has the precise of the unlawful equity and law implementation consultancy. With the expand in the utilization of the motorized frameworks to way offenses and smirch out illicits, PC information forecaster have begun giving their hands in serving the cops and investigators to flurry up the movement of breaking offenses. Criminal science is movement that is utilized to perceive offense and illegal qualities. The illicits and the offense rate plausibility can be evaluated with the assistance of criminal science strategies. The criminal science helps the police division, the investigator organizations and offense branches in distinguishing the genuine qualities of an unlawful. The criminal science territory has been worned in the records of offense path since the time 1800 . 
Offenses are a social aggravation and cost our overall population really severally. Without a doubt, the Indian Government has discovered a route approaches to make applications and programming for the use of State and Central Police regarding the Public Offense Records Office (NCRB).Any assessment that can help in tending to offenses faster will pay for itself. About $10 \%$ of the illicits submit about portion of the offenses. People who study criminal science will really need to perceive the illicits subject to the follows, characteristics and strategies for offense which can be assembled from the offense scene. In 1990s, data mining showed up as a strong instrument to remove supportive information from gigantic datasets and find the association between the credits of the data. Data mining at first came from bits of knowledge and man-made intelligence as an interdisciplinary field, anyway then it was grown an incredible arrangement that in 2001 it was considered as outstanding amongst other 10 driving advances which will change the world. As said previously, the Criminal science is a connection that hopes to perceive offense ascribes and it is conceivably the fundamental fields for applying data mining. By using this, data mining counts will really need to convey offense reports and help in the distinctive verification of illicits significantly snappier than any human could. Because of this surprising segment, there is a creating interest for data mining in criminal science. In actuality, Offense examination is a connection which consolidates researching the direct of the offenses, recognizing offenses and their relationship with illicits. The massive volume of offense and unlawful datasets and the unpredictability of associations between such information have made criminal science a fitting field for applying data mining strategies. Perceiving offense characteristics is the underlying advance for proceeding with any further examination. The idea of data examination depends essentially upon establishment data on analyst. An unlawful can go from regular infractions, for instance, illegal going to mental mistreatment mass crime like the 9/11 attacks, thusly it is difficult to exhibit the ideal count to cover all of them. The data that is gained from Data Mining approaches is an extraordinarily important and this can help and support, the police. Even more unequivocally, we can use gathering and grouping based models to help in distinctive evidence of offense plans and illicits. The wide extent of data mining applications in the criminal science has made it a critical field of investigation. Data mining structures have played as a basic occupation in aiding individuals in this quantifiable space and criminal science region. This makes it conceivably the most troublesome unique conditions for research. Lately, Enormous Information Investigation (BDA) has become an arising approach for examining information and extricating data and their relations in a wide scope of utilization territories. Because of ceaseless urbanization and developing populaces, states assume significant focal parts in our general public. To handle such issues, sociologists, investigators, and security foundations have given a lot of exertion towards mining likely examples and variables. According to public approach in any case, there are numerous difficulties in managing a lot of accessible information. Accordingly, new methodology and advances should be formulated to dissect this relevant data that would be heterogeneous and multi-sourced. The examination of such vast amounts of data enables us to adequately monitor happened occasions, recognize similitudes from episodes, send assets and settle on fast choices likewise. This could also help us gain a deeper understanding of both historical and current issues circumstances, at last guaranteeing improved wellbeing/security and personal satisfaction, just as expanded social and financial development. The quick development of distributed computing and information procurement and capacity advancements, from business and exploration establishments to governments and different associations, have prompted an immense number of exceptional degrees/intricacies from information that has been gathered and made freely accessible. It has gotten progressively critical to remove significant data and accomplish new experiences for understanding examples from such information assets. BDA can viably address BDA and 
Digging for Successful Perception and Patterns Estimating of Offense Information the difficulties of information that are excessively huge, excessively unstructured, and excessively quick to be overseen by conventional techniques. As a quickly developing and compelling practice, DBA can help associations to use their information and encourage new freedoms. Besides, BDA can be conveyed to help shrewd organizations push forward with more compelling tasks, high benefits and fulfilled clients. As a result, BDA is becoming increasingly important for associations to address their formative issues.Information mining is a creative, interdisciplinary, and developing exploration area as one of the key procedures of BDA, which can create standards and strategies across various fields for locating useful data and hidden examples in information. Information mining is useful not only for exploring different facts or marvels, but also for improving our understanding of old ones. Big Data Analytics can also facilitate us in defining offence patterns that occur in a specific destination and how they are associated to time using such techniques. The ramifications of AI and measurable procedures on offense or other enormous information applications, for example, auto collisions or time arrangement information, will empower the investigation, extraction and comprehension of related examples and patterns, eventually aiding offense counteraction and the board. In this paper, best in class AI and huge information examination calculations are used for the mining of offense information from three INDIAN, for example (Uttar Pradesh, New Delhi, Goa). Subsequent to pre-processing, containing information sifting and standardization, the high points are geo-planned utilising Google Maps to represent the beneficial results. Different methodologies in AI, profound learning, and time arrangement demonstrating are used for future patterns examination.

\section{ARCHITECTURE DIAGRAM}

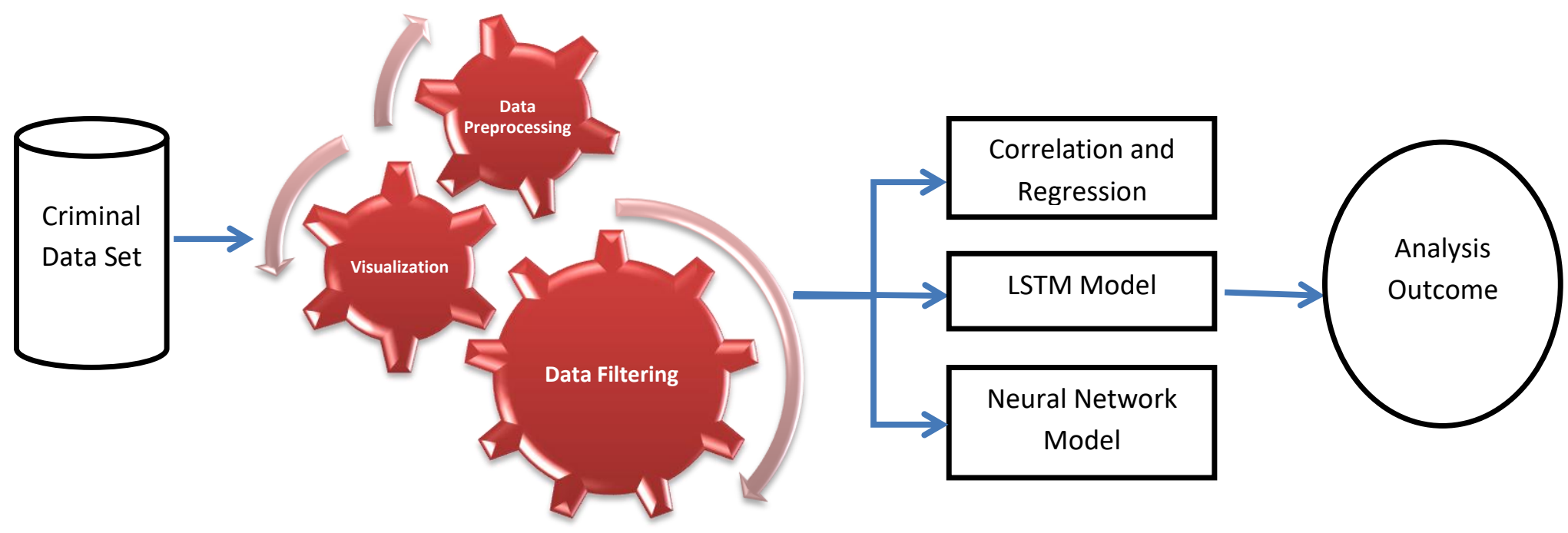

\section{LITERATURE SURVEY}

Fighting the bad behavior is ceaselessly been a necessity for the associations around the globe, different researches has been never really discover counter measures and markers of terrible conduct before occurring. BDA has become a discernible strategy for isolating and eliminating the data. Thusly, new methods and movements should be characterized to look at this different and various sourced information. 
By utilizing Geological appraisal, we can anticipate that that there are various techniques should plan regions of interest, yet among them, the choropleth organizing is broadly used to portray the geographic data of terrible conduct scenes.

What makes this cooperation more capable is the usage of latest and beneficial calculation. In this paper we will use Neural Organization Model, LSTM Model and Connection and Relapse.

\section{ANALYSIS AND VISUALIZATION OF DATA}

\section{A. BDA}

The three offence datasets we included in analysis are publicly available and cover three states in INDIA, for example (Uttar Pradesh, New Delhi, Goa). The Uttar Pradesh offense information contains offense occurrences from 01/01/2001 to 11/08/2010. Information from New Delhi has an absolute number of records, going back from 2001 to 2010. In the Goa dataset, there are offense occurrences which were caught from 01/01/2001 to 12/31/2010.

\section{B. DP}

Before performing any counts on our database, we perform the following data shaping preprocessing steps:

1) To consider time plan evaluating for the overall example within the data, time is discretized into a few areas.

2) We ascribed discretionary characteristics tried from the non-missing characteristics to some missing course credits in the New Delhi and Goa datasets, determined their mean, and afterward dislodged the missing ones.

3) The datetime of each offence shows the date and time of the event; we divided these credits into five highlights: Year (2001-2010), Month (1-12), Day (0-23), and Second (0-59).

\section{NARRATIVE REVELATION}

Considering the geographic contemplated the offense scenes, a characteristic guide subject to Google map was utilized for data portrayal, where offense occasions are gathered by their expansion/longitude information. As outlined in Fig. 1.

Figure 1 depicts the representation of offence practises on openstreet maps for the domains of (a)Uttar Pradesh, (b)New Delhi, and (c)Goa.

Figure 2 shows a time plan plot of offence events for the domains of (a) Uttar Pradesh, (b) New Delhi, and (c) Goa.

Figure 3. Top-6 offense cases for regions of (a)Uttar Pradesh, (b)New Delhi, and (c)Goa. As seen, blessing is a critical issue that bargains each state, close by some harsh offenses like Seizing and Mercilessness by Mate and Relatives.

Figure 4. Air pocket plot for street offense assessment: (a)Uttar Pradesh, (b)New Delhi, and (c)Goa related issues. New Delhi, in any case, is tortured by local and battery offense similarly as vehicle-related offenses. For Uttar Pradesh, the offense depiction is by and large 
essential, and the difficult issue of the city we found is related to Catching and Savagery by Life partner and Relatives.

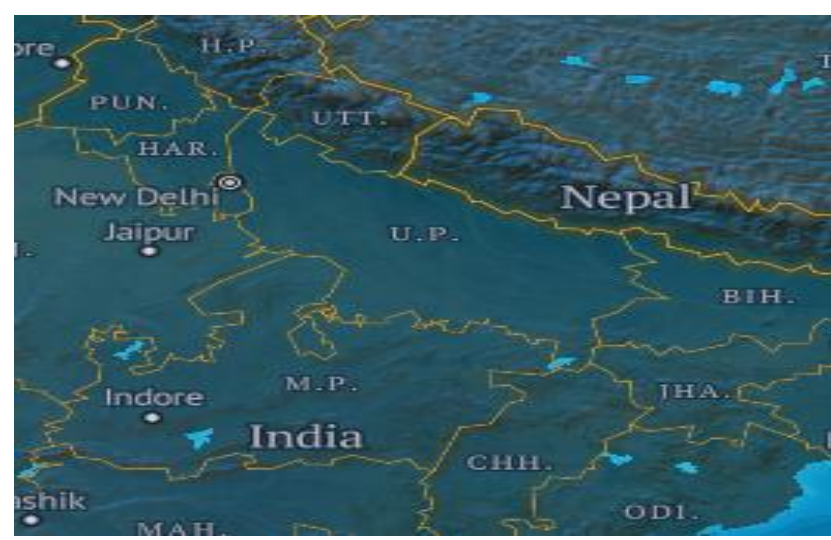

Figure 1 Visualization of criminal activity on open street maps for the states of (a) Uttar Pradesh, (b) New Delhi, and (c) Goa.

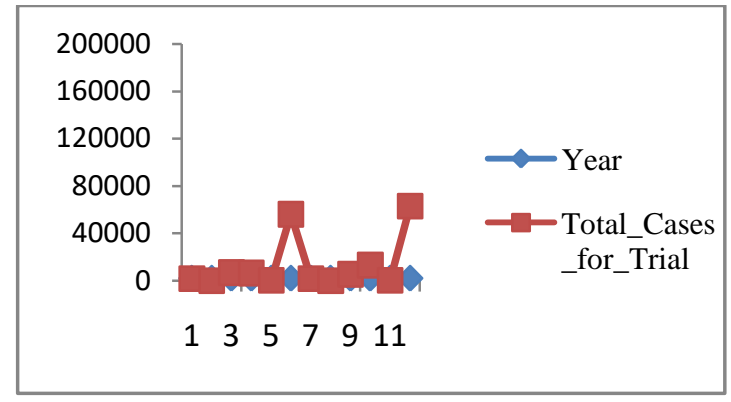

Figure 2 shows a time series plot of crime incidents for the states of (a) Uttar Pradesh, (b) New Delhi, and (c) Goa.

\section{CASES OF CRIMES}

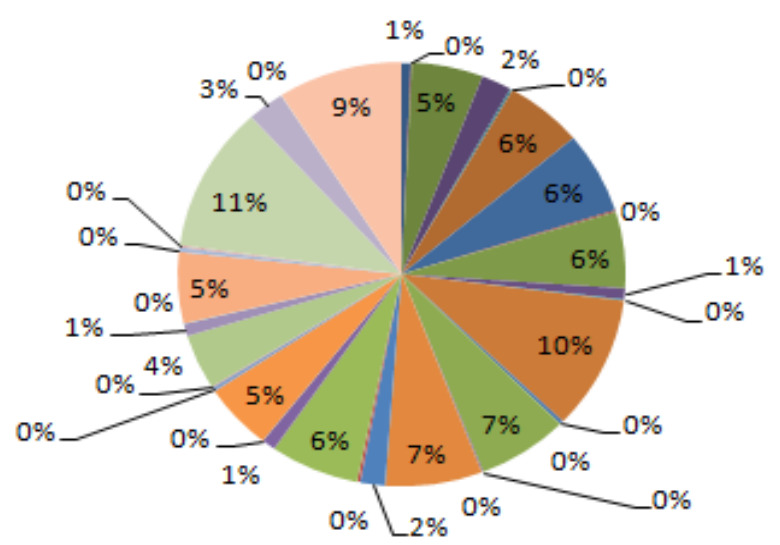

Figure 3 depicts the top six crime cases in the states of (a) Uttar Pradesh, (b) New Delhi, and (c) Goa 


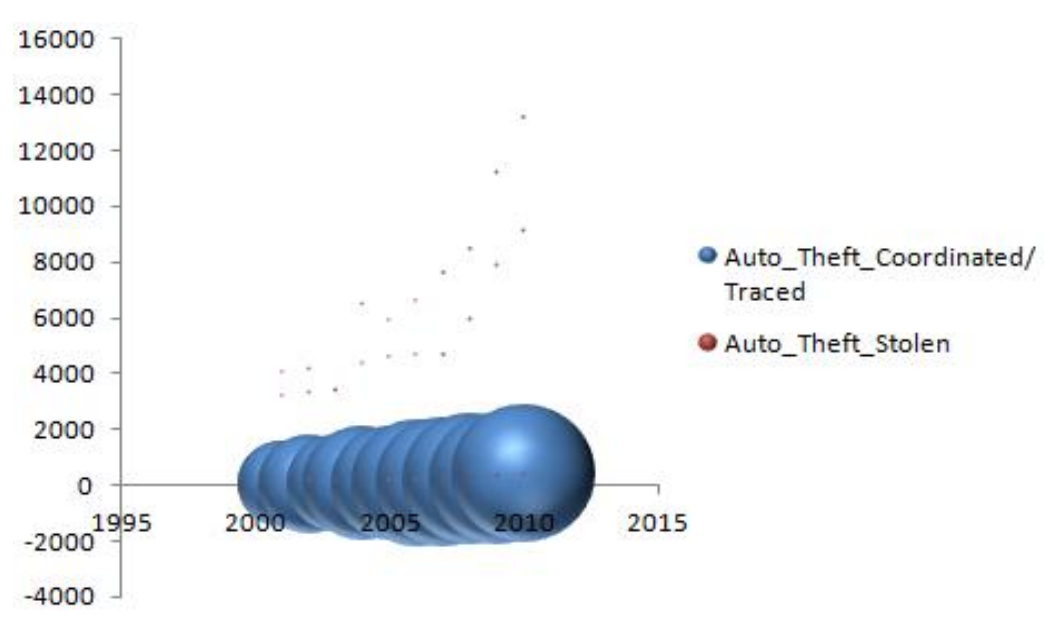

Figure 4 Analysis of street crime using a bubble plot: for states of (a)Uttar Pradesh, (b)New Delhi, and (c)Goa.

\section{THE FRAMEWORK FOR ALGORITHMS}

\section{A. NNM}

A neural organization is made out of a specific quantities of neurons, specifically hubs in the organization, which are coordinated in a few layers and associated with one another cross various layers. A neural organisation has at least three layers, for example, the information layer of perceptions, a non-recognizable secret layer in the centre, and a yield layer as the anticipated outcomes. We investigated the multi-faced feedforward network in this paper where each layer of hubs continues to receive commitments from the layer before it. The yields of a single layer's hubs will be used to contribute to the next layer.

$$
\begin{gathered}
\mathrm{zj}=\mathrm{bj}+\sum_{i=0}^{n} \text { wi j xi } \\
\mathrm{s}(\mathrm{z})=1 / 1+\mathrm{e}^{-\mathrm{z}}(2)
\end{gathered}
$$

\section{B. LONG SHORT TERM MEMORY MODEL}

This model is an amazing type of repetitive neural organisation that is capable of learning long-term conditions. For time course of action incorporates auto-association, for instance the presence of connection between's basically the time game plan and loosened types of itself, these are explicit useful in assumption because of their capacity of keeping up the state while seeing plans for the duration of the time game plan. The repetitive engineering empowers the states to be persevered, or impart between refreshed loads as every age advances. Besides, the model cell design can improve the RNN by empowering long haul diligence notwithstanding present moment.

$$
\begin{gathered}
\mathrm{f}(\mathrm{t})=\sigma\left(\mathrm{W}_{\mathrm{f}} \cdot\left[\mathrm{h}_{\mathrm{t}-1}, \mathrm{x}_{\mathrm{t}}\right]+\mathrm{b}_{\mathrm{f}}\right)(3) \\
\mathrm{i}_{\mathrm{t}}=\sigma\left(\mathrm{W}_{\mathrm{i}} \cdot\left[\mathrm{h}_{\mathrm{t}-1}, \mathrm{x}_{\mathrm{t}}\right]+\mathrm{b}_{\mathrm{i}}\right)(4) \\
\tilde{\mathrm{C}}=\tanh \left(\mathrm{W}_{\mathrm{C}} \cdot\left[\mathrm{h}_{\mathrm{t}-1}, \mathrm{x}_{\mathrm{t}}\right]+\mathrm{b}_{\mathrm{C}}\right)(5) \\
\mathrm{C}_{\mathrm{t}}=\mathrm{f}_{\mathrm{t}} * \mathrm{C}_{\mathrm{t}-1}+\mathrm{i}_{\mathrm{t}} * \widetilde{\mathrm{C}}(6)
\end{gathered}
$$




\section{EXPRRIMENTAL RESULTS}

\section{A. ANALYSIS OF RESULT}

To estimate offence patterns, we investigated deep learning calculations and time arrangement gauge models. For show assessment, the Root Mean and spearman relationship are utilized in specifications of assorted limitations and different sizes of guidance tests. The above relationship are characterized as follows. We assessed the presentation of the expectation models while changing the quantity of preparing a long time from 1 to 10 and the outcomes are summed up in Table 1.

\begin{tabular}{|c|l|l|}
\hline States & $\begin{array}{c}\text { Neural } \\
\text { Network }\end{array}$ & $\begin{array}{c}\text { Corelation } \\
\text { LSTM }\end{array}$ \\
\hline DELHI & 0.09 & 0.99 \\
\hline GOA & 0.36 & 0.94 \\
\hline UTTAR & 0.42 & 0.67 \\
PRADESH & & \\
\hline
\end{tabular}

TABLE 1: Similarity of various algorithms/models in the preceding provisions under diverse samples.

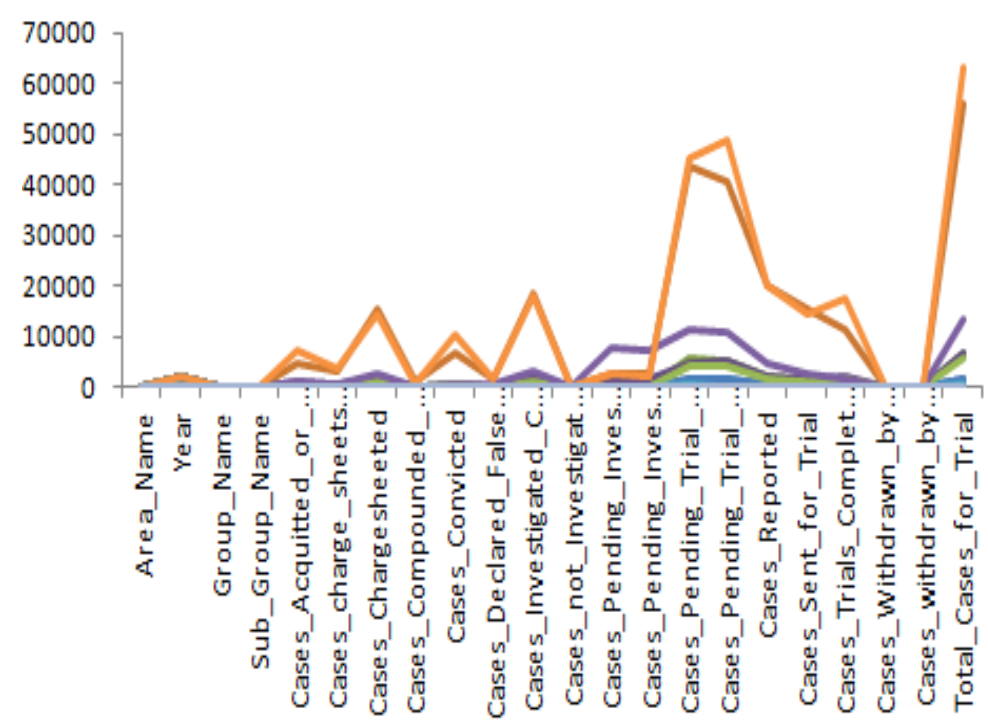

The outcomes likewise showed that LSTM model performed better compared to customary neural organization models as illustrated in table. 1 that neural network seems has lower RMSE but the correlation between predicted values and the real ones is low.

\section{CONCLUSION \& FUTURE WORK}

Huge information is an element of information science that disconnect assorted approaches to decision, methodicallly separate realities from educational aggregation that are horribly gigantic or complex to be overseen by standard data taking care of use programming. In this paper a progression of best in class huge information investigation and representation 
procedures were used to break down offense huge information from three INDIAN states, which permitted us to distinguish designs and acquire patterns. By investigating a neural organization model, and the profound learning calculation LSTM, we came to a decision that the long short term memory calculation perform better compared to regular neural organization models. Extra outcomes clarified before will give new experiences into offense drifts and will benefit both police departments and law enforcement officials and agencies in their dynamic. In future we intend to consolidate multivariate perception to reveal more expected examples and patterns inside these datasets.

\section{REFERENCES}

[1] M. Huda, A. Maseleno, M. Siregar, R. Ahmad, K. A. Jasmi, N. H. N. Muhamad, and P. Atmotiyoso, "Big data emerging technology: Insights into innovative environment for online learning resources,' Int.J. Emerg. Technol. Learn., vol. 13, no. 1, pp. 23-36, Jan. 2018.

[2] J. Zakir and T. Seymour, “Big data analytics,' Issues Inf. Syst., vol. 16, no. 2, pp. 81-90, 2015.

[3] J. Zhao, Y. Gao, Y. Qu, H. Yin, Y. Liu, and H. Sun, “Travel time prediction: Based on gated recurrent unit method and data fusion," IEEE Access, vol. 6, pp. 70463-70472, 2018.

[4] J. Peral, A. Ferrández, D. Gil, E. Kauffmann, and H. Mora, "A review of the analytics techniques for an efficient management of online forums: An architecture proposal," IEEE Access, vol. 7, pp. 12220-12240, 2019.

[5] K. Niu, H. Zhang, C. Cheng, C. Wang, and T. Zhou, "'A novel spatiotemporal model for city-scale traffic speed prediction," IEEE Access, vol. 7, pp. 30050-30057, 2019.

[6] L. Guo, X. Cai, F. Hao, D. Mu, C. Fang, and L. Yang, "Exploiting finegrained coauthorship for personalized citation recommendation,' IEEE Access, vol. 5, pp. 1271412725, 2017.

[7] L. Guo, X. Cai, F. Hao, D. Mu, C. Fang, and L. Yang, "Exploiting finegrained coauthorship for personalized citation recommendation,' IEEE Access, vol. 5, pp. 1271412725, 2017.

[8] L. Stopar, P. Skraba, D. Mladenic, and M. Grobelnik, "StreamStory: Exploring multivariate time series on multiple scales,' IEEE Trans. Vis. Comput. Graphics, vol. 25, no. 4, pp. 1788-1802, Apr. 2019.

[9] L. Stopar, P. Skraba, D. Mladenic, and M. Grobelnik, "StreamStory: Exploring multivariate time series on multiple scales,' IEEE Trans. Vis.Comput. Graphics, vol. 25, no. 4, pp. 1788-1802, Apr. 2019.

[10] S. Zheng, S. Chen, L. Yang, J. Zhu, Z. Luo, J. Hu, and X. Yang, “Big data processing architecture for radio signals empowered by deep learning: Concept, experiment, applications and challenges,"' IEEE Access, vol. 6, pp. 55907-55922, 2018.

[11] U. Thongsatapornwatana, "A survey of data mining techniques for analyzing crime patterns," in Proc. 2nd Asian Conf. Defence Technol., Chiang Mai, Thailand, 2016, pp. $123-128$.

[12] Y. Gao, Y. Xia, J. Qiao, and S. Wu, "Solution to gang crime based on graph theory and analytical hierarchy process,'” Neurocomputing, vol. 140, pp. 121-127, Sep. 2014. 